\title{
The relationship between metabolic syndrome and albuminuria in patients with type 2 diabetes mellitus
}

\section{Osman Z Sahin ${ }^{1}$, Serap B Sahin², Teslime Ayaz ${ }^{3}$ \\ ${ }^{1}$ Recep Tayyip Erdogan University Medical School, Department of Nephrology, Rize, Turkey \\ 2 Recep Tayyip Erdogan University Medical School, Department of Endocrinology and Metabolism Disease, Rize, Turkey \\ ${ }^{3}$ Recep Tayyip Erdogan University Medical School, Department of Internal Medicine, Rize, Turkey}

\section{Objectives:}

\section{Methods:}

\section{Results:}

Conclusions.

Obesity, as a central piece inside metabolic syndrome, is as an independent risk factor for the onset, aggravated course, and poor outcomes of chronic kidney disease (CKD) including diabetic nephropathy (DN). Our aim was to investigate the relationship between metabolic syndrome (MS), diabetic nephropathy and renal function in patients with type 2 diabetes mellitus (T2DM).

197 patients with T2DM (mean age; $56.1 \pm 10.2$ years, female/male; 127/70) were enrolled. NCEP ATP III criteria was used for the diagnosis of MetS. Albuminuria and estimated glomerular filtration rate (eGFR) were measured.

$79.3 \%$ of the patients had MS. HbA1c levels were similar in patients with (8.5 \pm 2.2 vs $8.5 \pm 2.1)$ and without MS $(p=0.888)$. The frequencies of diabetic retinopathy and neuropathy were not different in patients according to the presence of MS ( $p>0.05)$. Prevalence of microalbuminuria $(32.2 \%$ vs $46 \%)$ and macroalbuminuria $(12.3 \%$ vs $7.7 \%)$ was similar in subjects with and without MS $(p=0.248)$. We did not find a decrease in eGFR in patients with MS compared to the patients without MS. While uric acid levels were higher in patients with MS $(5.5 \pm 1.5$ vs $4.7 \pm 1.5 \mathrm{mg} / \mathrm{dl}$, $p=0.006)$, hsCRP values did not differ $(p=0.689) .72 .4 \%$ of the subjects were obese and the prevalence of albuminuria was not different in obese and nonobese patients $(p=0.596)$.

In this study we did not find an association between obesity, MS and DN. 